\title{
Real-Time Quantitative Elasticity Imaging of Deep Tissue Using Free-Hand Conventional Ultrasound
}

\author{
Ali Baghani ${ }^{1}$, Hani Eskandari ${ }^{1}$, Weiqi Wang ${ }^{1}$, \\ Daniel Da Costa ${ }^{1}$, Mohamed Nabil Lathiff ${ }^{1}$, Ramin Sahebjavaher ${ }^{1}$, \\ Septimiu Salcudean ${ }^{1}$, and Robert Rohling ${ }^{1,2}$ \\ 1 Department of Electrical and Computer Engineering, University of British \\ Columbia, Vancouver, BC, Canada \\ baghani@ece.ubc.ca \\ 2 Co-appointed with the Department of Mechanical Engineering, \\ University of British Columbia, Vancouver, BC, Canada
}

\begin{abstract}
In this article an ultrasound elastography technology is reported which provides quantitative images of tissue elasticity from deep soft tissue. The technique is analogous to Magnetic Resonance Elastography in the use of external mechanical vibrations which can penetrate deep tissue. Multifrequency steady-state mechanical vibrations are applied to the tissue at the skin and tissue displacements are measured by a conventional ultrasound system. Absolute values of tissue elasticity are computed in real-time for each frequency and displayed to the physician. The quantitative elasticity images produced by the technology are validated with magnetic resonance elastography images as the gold standard on standard elasticity phantoms. Preliminary in-vivo data from healthy volunteers are presented which show the potential of the technology for clinical use. The system is currently being used in different clinical studies to image kidney fibrosis, liver fibrosis, and prostate cancer.
\end{abstract}

\section{Introduction}

Elastography has emerged as an imaging modality providing new information to the clinician about the mechanical properties of tissue [1. It has found a place in the imaging of breast lesions [2, liver fibrosis [3], and is being investigated in many other clinical areas such as targeting of prostate cancer for biopsy and focal therapy [4.

The first generation of elastography technology was developed on ultrasound machines [5] and created images of relative elasticity of tissue. The clinician applied a manual compression with the probe to deform the tissue, and the elastography system measured the tissue strain. Under certain assumptions, the tissue strain is inversely proportional to the tissue stiffness, and therefore strain images can show the contrast in tissue stiffness. The first generation elastography 
is now available on many medium to high-end ultrasound systems under different brand names such as elastography, strain imaging, real-time elastography, etc.

The general trend in radiology towards quantitative imaging, together with the difficulties in applying the manual compression, called for more innovation and through the efforts of different groups, second generation elastography technologies were born [6, 7. A second generation elastography technique creates quantitative elasticity images where the contrast in the image is the absolute elasticity of the tissue.

Elastography has been developed primarily with ultrasound or magnetic resonance imaging [8] as the underlying imaging device to track tissue displacements. To cause tissue displacements, different methods have been devised with mechanical transient excitation [2, mechanical steady-state excitation [8, 9], and acoustic radiation force (shear wave) transient excitation 6, 7. to name a few.

The majority of the magnetic resonance imaging techniques use a steadystate mechanical excitation to image the tissue elasticity [8, 10, 11]. The idea is to measure the wavelength of the steady-state wave patterns in the tissue from which the wave speed can be estimated. The wave speed depends on the mechanical properties of tissue, and is generally higher in stiffer tissue compared to softer tissue.

The reported technology uses analogous techniques as used in magnetic resonance elastography for ultrasound elastography. A steady-state mechanical vibration is applied to the tissue while the tissue is imaged by the ultrasound. From the sequence of ultrasound images, the tissue displacements and wave patterns are computed. The local wavelength of the wave pattern is then estimated to create a map of the tissue stiffness which is displayed in real-time.

This article gives an overview of the technology and reports the most recent advances. In particular we report the first direct comparison of the technique with MRE on a standard quality assurance phantom. Steady-state excitation was first used in sonoelasticity to image tissue stiffness [12. It has also been used before to produce MRE-type elasticity images [13. However this is the first report of an implementation for "real-time" operation with "free-hand" conventional ultrasound. Two novel ideas which have enabled these advances are the use of a "thin-slice" consisting of a few planes for 3D data acquisition, and the fast implementation of all the image processing pipeline on a graphics processing unit (GPU). These advances are reported for the first time in this article. Based on these qualities, the technology holds promise for ultrasound guided procedures, such as biopsies, by providing additional quantitative information to the clinician.

\section{Methods}

The system has been implemented on a SonixTouch platform (Ultrasonix Medical Corp., Richmond, BC, Canada) (Fig. 1 (a)). Two prototype systems have been developed based on the Texo and Ulterius software development kits. A mechanical vibration source (LDS V203, LDS/B\&K, Norcross, GA) has been 
mounted on the flexible arm of the SonixTouch. The hardware of the system has been upgraded with a CUDA supporting graphics card (GTX 580 GPU, NVIDIA, Santa Clara, CA). The SonixTouch collects the RF-data which are then transferred to the memory of the graphics card. All the computational algorithms for absolute elastography have been implemented in CUDA and run on the GPU in real-time. The absolute elasticity images are transferred back to the CPU memory and displayed.

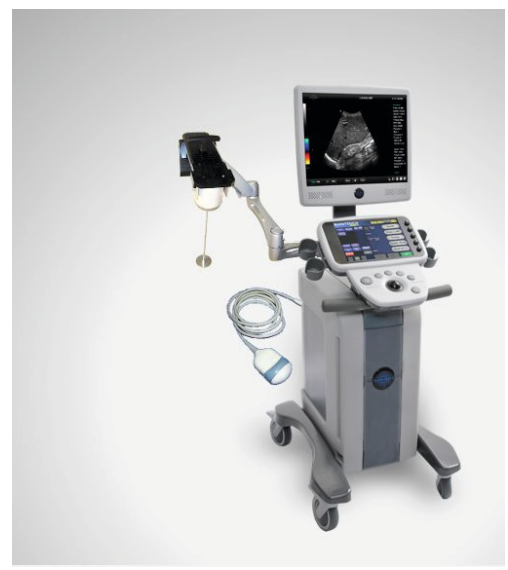

(a)

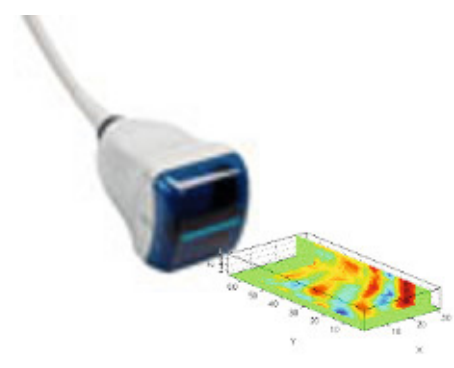

(b)

Fig. 1. (a) A photo of the actual elastography system with the vibration source mounted on the SonixTouch arm (b)The 4DL14-5 3D probe and the thin volume of displacement data acquired from 7 planes

The image processing pipeline of the system is shown in Fig. 2 The RF-data are processed by the speckle tracking block to find the time-domain displacements, which are then converted to frequency-domain displacements or phasors by a Fourier transform for each frequency in the excitation. The phasors are then passed through the inversion algorithm. An algorithm used for the speckle tracking is described in 14 and different algorithms for the inversion are compared in [15]. Here we used the Local Frequency Estimation method [8]. Average computational times are compared in table 1 for CPU vs. GPU on test data sets.

The system can be used with 2D and 3D probes to perform absolute elastography. With 2D probes, the variations of the waves in the elevational direction cannot be observed and measured. As a result, the elasticity images obtained with $2 \mathrm{D}$ probes suffer some over-estimation in the elasticity values (10 to 60

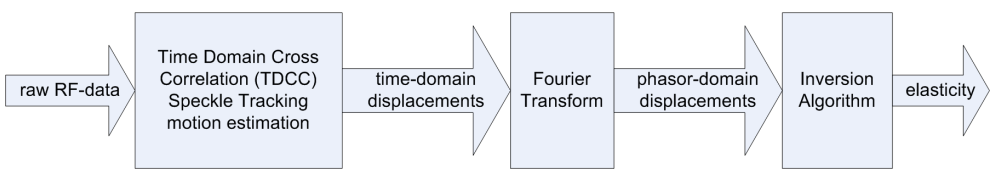

Fig. 2. Image processing pipe-line 
Table 1. Comparison of computational time between CPU and GPU implementations of the elastography algorithms

\begin{tabular}{c|c|c}
\hline & $\begin{array}{c}\text { CPU time (ms) } \\
\text { Core i5 660@3.33GHZ }\end{array}$ & $\begin{array}{c}\text { GPU time (ms) } \\
\text { GTX 480 }\end{array}$ \\
\hline Speckle Tracking & 1600 & 50 \\
\hline Fourier Transform & 110 & 9 \\
\hline Inversion & 1800 & 12 \\
\hline
\end{tabular}

percent is typical). The error has some dependency on the placement of the vibration source. The major benefit of $2 \mathrm{D}$ probes, however, is the faster acquisition of US data which results in a higher frame rate.

The elastograms obtained with 3D probes are more accurate and robust, but they suffer from a lower frame rate. A thin volume (typically consisting of 5 to 7 planes) is acquired to capture the variation of the wave in the elevational direction (see Fig. 1(b)). Currently the system generates elasticities at a rate of 1 image every 2 seconds with a 4DL14-53D probe and 80mm depth of imaging.

Fig. 3 shows variation of the wave pattern (real part of the displacement phasor) in the elevational direction as measured by a 3D probe in two different configurations. By comparing the two cases, it can be observed that by changing the placement of the excitation source relative to the probe, the variation of the displacement in the elevational direction can be minimized. In this case a relatively accurate estimate of the elasticity can be obtained by using a $2 \mathrm{D}$ probe.

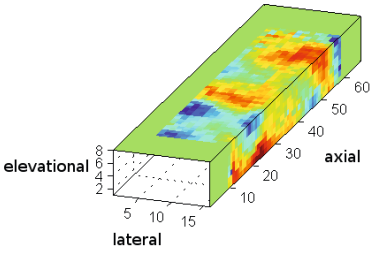

(a)

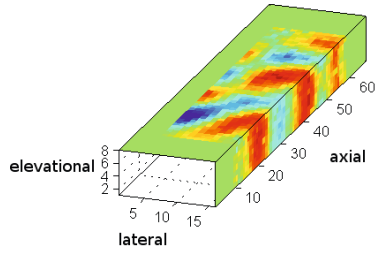

(b)

Fig. 3. The variation of displacement in the elevational direction depends on the placement of the excitation source (a) more variation (b) less variation in the elevational direction

\section{Validation with MRE}

The mechanism of creating waves in the tissue by steady-state mechanical vibration is analogous in the reported system and MRE. The methods which are used to invert the wave patterns and compute the elasticities are also analogous. Therefore, one would expect that an absolute elastogram produced by the reported system should have the same appearance and values as an MRE absolute elastogram, as a gold standard. 
To prove this hypothesis, experiments were performed with a standard elasticity phantom; The elasticity quality assurance phantom model 049 (CIRS Inc, Norfolk, VA, USA) was imaged using a 3T Achieva MRI system (Philips Inc, Netherlands). This phantom includes different spherical inclusions of varying stiffness and sizes. The frequency of excitation was $200 \mathrm{~Hz}$. The elastogram obtained for a horizontal plane passing through the larger inclusions is shown in Fig 4(a).

The same phantom was imaged using a 4DL14-5 linear 3D probe (Ultrasonix Medical Corp., Richmond, BC, Canada) with the system. The probe was mounted on a linear stage and moved along the long axis of the phantom to image a larger area covering multiple inclusions. The vibration was applied with the arm mounted voice coil of the system at $200 \mathrm{~Hz}$. At each location of the linear stage, a slim volume of ultrasound data was acquired and used to reconstruct a single plane of elasticity (mid-plane of the slim volume). By moving the 3D probe to a new position, another elasticity plane was produced and so forth. By putting these single plane elasticity images together a whole volume elasticity map was produced. The result is shown in Fig. 4(b). A comparison of Figs 4 (a) and 4(b) provides some evidence for the validity of hypothesis. The elasticity values obtained from the two methods are reported in table 2 as well as the manufacturer values.

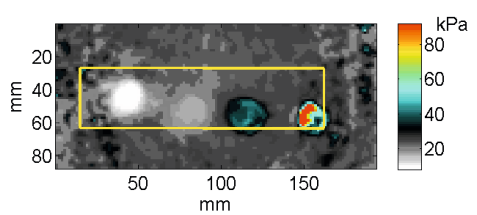

(a)

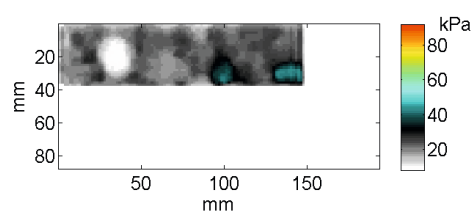

(b)

Fig. 4. (a) Magnetic Resonance Elastography and (b) Ultrasound elastography images acquired by the system

Table 2. Comparison of average elasticity values between MRE, ultrasound elastography, and manufacturer values

\begin{tabular}{c|c|c|c}
\hline & MRE $(\mathrm{kPa})$ & $\mathrm{US} / \mathrm{E}(\mathrm{kPa})$ & Man. $(\mathrm{kPa})$ \\
\hline Softest Inclusion & 8 & 9.5 & 6 \\
\hline Soft Inclusion & 17 & 17 & 17 \\
\hline Hard Inclusion & 42 & 40 & 54 \\
\hline Hardest Inclusion & 54 & 45 & 62 \\
\hline Background & 24 & 24 & 29 \\
\hline
\end{tabular}

\section{In Vivo Results}

The system has been used to image different tissue in-vivo and ex-vivo with different probes. In this section, a sample of the data collected so far is reported. 
The 4DL14-5 linear 3D transducer was used to image the liver of consenting healthy volunteers in-vivo to a depth of $80 \mathrm{~mm}$. The transducer was placed on the side of the patient and images were obtained intercostally. A multi-frequency excitation, consisting of 50,80 , and $100 \mathrm{~Hz}$ components was applied via the external vibrator. As with MRE studies, we observe that the elasticity increases as a function of frequency [16. The trend can be seen in the elastograms of a volunteer, at the three frequencies, shown in Fig. 5. In all the images, a stiffer layer corresponding to intercostal muscle can be observed near the skin, while the liver has a fairly homogeneous appearance in the elastogram.
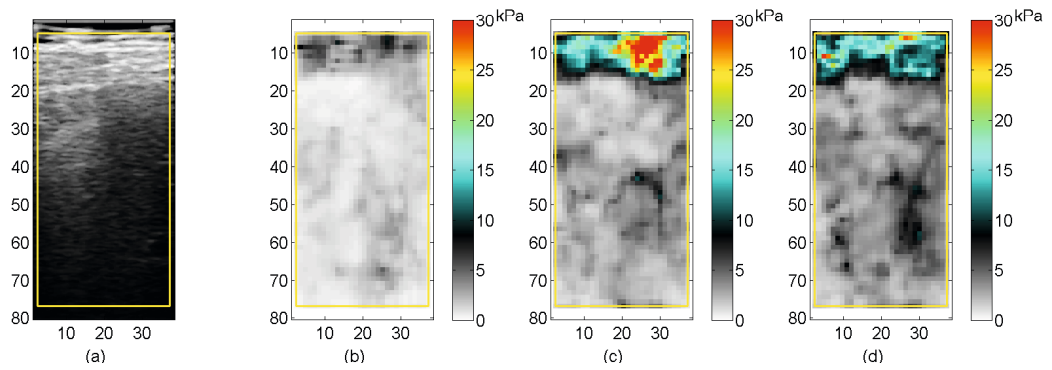

Fig. 5. The frequency dependence of elasticity from a healthy volunteer liver (a) Bmode image (b) elastogram at $50 \mathrm{~Hz}$, (c) elastogram at $80 \mathrm{~Hz}$, (d) elastogram at 100 $\mathrm{Hz}$
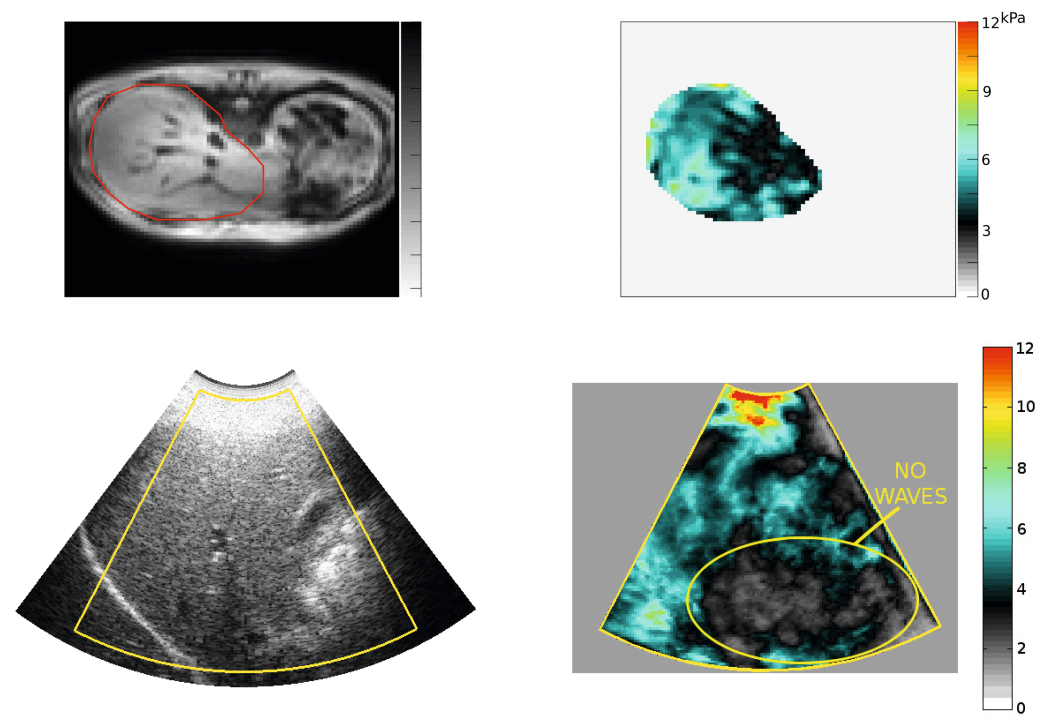

Fig. 6. Top: MRI image and MRE image at $56 \mathrm{~Hz}$, Bottom: B-Mode and elastogram from a healthy volunteer liver averaged between 55 and $60 \mathrm{~Hz}$, obtained with a low frequency curved 3D transducer 
In another experiment, a comparison was made between MRE and the proposed system. The 4DC7-3 curved transducer was used to image the liver of a consenting healthy volunteer at $55 \mathrm{~Hz}$ and $60 \mathrm{~Hz}$ down to a depth of $150 \mathrm{~mm}$. The transducer was placed on the side of the volunteer and images were obtained intercostally. The B-mode and average elasticity images are shown in Fig. 6 as well as the MRE data of the same volunteer collected at $56 \mathrm{~Hz}$. The range of values observed are similar in both elastograms. However, a more carefully designed experiment is needed with registration of the ultrasound and MRI images before a conclusion can be made.

\section{Conclusion}

In this article an ultrasound elastography technology is reported which is analogous to magnetic resonance elastography in the type of excitation and inversion algorithms used. The absolute elasticity values obtained by the system are validated against MRE values as a gold standard on a quality assurance elasticity phantom. At low frequencies $(30-100 \mathrm{~Hz})$ the mechanical vibrations can penetrate deep tissue, and provide the necessary displacements for measurement of tissue elasticity at depths of $150 \mathrm{~mm}$. Typical in vivo elastograms obtained by imaging healthy volunteer livers with different transducers are reported. The values reported are similar to the values reported in the literature from MRE studies.

\section{References}

1. Lerner, R., Parker, K., Holen, J., Gramiak, R., Waag, R.: Sono-elasticity: Medical elasticity images derived from ultrasound signals in mechanically vibrated targets. Acoustical Imaging 16, 317-327 (1988)

2. Bercoff, J., Chaffai, S., Tanter, M., Sandrin, L., Catheline, S., Fink, M., Gennisson, J.L., Meunier, M.: In vivo breast tumor detection using transient elastography. Ultrasound Med. Biol. 29(10), 1387-1396 (2003)

3. Huwart, L., Peeters, F., Sinkus, R., Annet, L., Salameh, N., ter Beek, L.C., Horsmans, Y., Beers, B.E.V.: Liver fibrosis: Non-invasive assessment with MR elastography. NMR in Biomedicine 19, 173-179 (2006)

4. Lorenz, A., Sommerfeld, H., Garcia-Schurmann, M., Philippou, S., Senge, T., Ermert, H.: A new system for the acquisition of ultrasonic multicompression strain images of the human prostate in vivo. IEEE Transactions on Ultrasonics, Ferroelectrics and Frequency Control 46(5), 1147-1154 (1999)

5. Ophir, J., Cespedes, I., Ponnekanti, H., Yazdi, Y., Li, X.: Elastography: A quantitative method for imaging of elasticity of biological tissues. Ultrasonic Imaging 13, 111-134 (1991)

6. Bercoff, J., Tanter, M., Fink, M.: Supersonic shear imaging: A new technique for soft tissue elasticity mapping. IEEE Trans. Ultrason. Ferroelect. Freq. Contr. 51(4), 396-409 (2004)

7. Nightingale, K., Palmeri, M., Nightingale, R., Trahey, G.: On the feasibility of remote palpation using acoustic radiation force. J. Acoust. Soc. Am. 110(1), 625-634 (2001) 
8. Muthupillai, R., Lomas, D., Rossman, P., Greenleaf, J., Manduca, A., Ehman, R.: Magnetic resonance elastography by direct visualization of propagating acoustic strain waves. Science 269(5232), 1854-1857 (1995)

9. Baghani, A., Brant, A., Salcudean, T., Rohling, R.: A high frame rate ultrasound system for the study of tissue motions. IEEE Trans. Ultrason. Ferroelect. Freq. Contr. 57(7), 1535-1547 (2010)

10. Sinkus, R., Tanter, M., Xydeas, T., Catheline, S., Bercoff, J., Fink, M.: Viscoelastic shear properties of in vivo breast lesions measured by MR elastography. Magnetic Resonance Imaging 23, 159-165 (2005)

11. Asbach, P., Klatt, D., Hamhaber, U., Braun, J., Somasundaram, R., Hamm, B., Sack, I.: Assessment of liver viscoelasticity using multifrequency MR elastography. Magnetic Resonance in Medicine 60(2), 373-379 (2008)

12. Lerner, R., Huang, S., Parker, K.: Sonoelasticity images derived from ultrasound signals in mechanically vibrated tissues. Ultrasound in Medicine and Biology 16(3), 231-239 (1990)

13. Muller, M., Gennisson, J., Deffieux, T., Sinkus, R., Annic, P., Montaldo, G., Tanter, M., Fink, M.: Full 3D inversion of the viscoelasticity wave propagation problem for 3D ultrasound elastography in breast cancer diagnosis. In: IEEE Ultrasonics Symposium, pp. 672-675. IEEE (2007)

14. Zahiri-Azar, R., Salcudean, S.: Motion estimation in ultrasound images using time domain cross correlation with prior estimates. IEEE Transactions on Biomedical Engineering 53(10), 1990-2000 (2006)

15. Baghani, A., Salcudean, S., Honarvar, M., Sahebjavaher, R., Rohling, R., Sinkus, R.: Travelling wave expansion: A model fitting approach to the inverse problem of elasticity reconstruction. IEEE Transactions on Medical Imaging 30(8), 1555-1565 (2011)

16. Asbach, P., Klatt, D., Schlosser, B., Biermer, M., Muche, M., Rieger, A., Loddenkemper, C., Somasundaram, R., Berg, T., Hamm, B., et al.: Viscoelasticitybased staging of hepatic fibrosis with multifrequency MR elastography. Radiology $257(1), 80-86(2010)$ 